\title{
Parasitoides, Braconidae (Hymenoptera) y Tachinidae (Diptera) de barrenadores, Crambidae y Coleophoridae (Lepidoptera) de caña de azúcar para la producción de panela en Colombia
}

\author{
Parasitoids, Braconidae (Hymenoptera) and Tachinidae (Diptera) of borers, Crambidae \\ and Coleophoridae (Lepidoptera) of sugarcane for the production of panela in Colombia
}

\author{
(iD ZAIDA XIOMARA SARMIENTO-NAIZAQUE ${ }^{1 *}$; ID CARLOS E. \\ SARMIENTO $^{2}$; iD NANCY BARRETO-TRIANA ${ }^{1}$
}

\footnotetext{
${ }^{1 *}$ Corporación Colombiana de Investigación Agropecuaria (Agrosavia), Centro de Investigación Tibaitatá, Cundinamarca, Colombia,zsarmiento@agrosavia.co, nbarreto@agrosavia.co. ${ }^{2}$ Universidad Nacional de Colombia. Instituto de Ciencias Naturales, Bogotá, Colombia, cesarmientom@unal.edu.co.
}

\begin{abstract}
*Autor para correspondencia
Zaida Xiomara Sarmiento-Naizaque Corporación Colombiana de Investigación Agropecuaria (Agrosavia), Centro de Investigación Tibaitatá, km 14 vía Mosquera, Cundinamarca, Colombia, zsarmiento@agrosavia.co.

Citación sugerida

SARMIENTO-NAIZAQUE Z. X., SARMIENTO, C. E.; BARRETO-TRIANA, N. 2021. Parasitoides, Braconidae (Hymenoptera) y Tachinidae (Diptera) de barrenadores, Crambidae y Coleophoridae (Lepidoptera) de caña de azúcar para la producción de panela en Colombia. Revista Colombiana de Entomología 47 (2): e10558. https://doi.org/10.25100/socolen. v47i2.10558
\end{abstract}

Recibido: 19-Ago-2020

Aceptado: 12-Feb-2021

Publicado: 15-Oct-2021

Revista Colombiana de Entomología ISSN (Print): 0120-0488

ISSN (On Line): 2665-4385

https://revistacolombianaentomologia.univalle.edu.co

Open access

(c) (i) (5) (2) BY-NC-SA 4.0

Publishers: Sociedad Colombiana de Entomología SOCOLEN (Bogotá, D. C., Colombia) https://www.socolen.org.co

Universidad del Valle (Cali, Colombia)

https://www.univalle.edu.co

(C) 2021 Sociedad Colombiana de Entomología SOCOLEN y Universidad del Valle - Univalle
Resumen: En los agroecosistemas de caña de azúcar hay enemigos naturales que pueden regular las poblaciones de los barrenadores (Lepidoptera), este rol es primordial considerando que el control biológico es la principal estrategia para el manejo. El objetivo de esta investigación fue proveer una lista actualizada de los parasitoides de los barrenadores de caña de azúcar para panela de la Hoya del río Suárez (Colombia). Se revisaron 616 especímenes parasitoides obtenidos entre 2015 y 2017 a partir de 4108 larvas recolectadas en cultivos de caña de azúcar con el síntoma de corazón muerto. De las diez especies encontradas Alabagrus albispina, A. imitatus, A. parvifaciatus, A. roibasi (Hymenoptera: Braconidae) y Leskia sp. (Diptera: Tachinidae) son nuevos registros para Colombia. Alabagrus albispina, A. roibasi y Leskia sp. son registros nuevos para los barrenadores del cultivo. Estas especies pueden servir como nuevas herramientas para el control biológico en caña de azúcar.

Palabras clave: Enemigos naturales, Braconidae, Tachinidae, cultivo de caña, Hoya del río Suárez, agentes de control biológico.

Abstract: Sugarcane agroecosystems include natural enemies that can regulate the populations of stemborers (Lepidoptera), and this is an important role considering that biological control is the main management strategy for the crop. The goal of this research was to offer an updated list of stemborer parasitoids of sugarcane for panela from the Suárez river basin (Colombia). A total of 616 parasitoid specimens were sampled from 2015 to 2017. Out of the ten species found Alabagrus albispina, A. imitatus, A. parvifaciatus, A. roibasi (Hymenoptera: Braconidae) and Leskia sp. (Diptera: Tachinidae) are new records for Colombia. Alabagrus albispina, A. roibasi, and Leskia sp. are new records for the stem borers of the crop. These species may serve as new tools for biological control in sugarcane.

Keywords: Natural enemies, Braconidae, Tachinidae, sugarcane crop, Suárez river basin, biological control agents.

\section{Introducción}

En Colombia, los barrenadores de tallo del género Diatraea se consideran las plagas más importantes del cultivo de caña de azúcar (Saccharum officinarum L.) (Bustillo 2011; Vargas et al. 2015). Particularmente, en la región de la Hoya del río Suárez ubicada en los departamentos de Boyacá y Santander, una de las principales zonas productoras de caña de azúcar para panela en el país, se han identificado algunas especies del complejo, como D. saccharalis (Fabricius, 1974), D. busckella Dyar \& Heinrich, 1927, Diatraea pos. rosa y otros barrenadores como Eoreuma insuastii Solis \& Osorio-Mejía, 2020 (Lepidoptera: Crambidae) y Blastobasis sp. (Lepidoptera: Coleophoridae) (Sandoval-Cáceres et al. 2016; Solis et al. 2020).

El control biológico es la herramienta principal para manejar los barrenadores del tallo del género Diatraea en Colombia (Vargas et al. 2015). Posada y García (1976) en un listado mencionaron varias especies parasitoides de Diatraea como las moscas de la familia Tachinidae: Billaea claripalpis (Wulp, 1896), Lydella minense (Townsend, 1927), Genea jaynesi (Aldrich, 1932) y la avispa de la familia Braconidae: Alabagrus stigma (Brullé, 1846). Gómez et al. (1996), señalaron al parasitoide 
de huevos Trichogramma exiguum Pinto y Platner, 1978 (Hymenoptera: Trichogrammatidae) como una especie ideal en el control de Diatraea y Bustillo (2011) adicionó a Cotesia flavipes Cameron, 1891 (Hymenoptera: Braconidae), que ataca larvas de Diatraea spp. y a Phytomyptera sp. como parasitoide del barrenador menor Blastobasis graminea Adamski, 1999.

En varios países de América se han empleado exitosamente los parasitoides T. exiguum (Hymenoptera: Trichogrammatidae), C. flavipes (Hymenoptera: Braconidae), $L$. minense y B. claripalpis (Diptera: Tachinidae) en el control de Diatraea spp. (Leyton-Flor et al. 2018; Weir et al. 2007; Parra et al. 2010; Borges et al. 2019).

Bustillo (2011) señaló doce parasitoides que ejercen control sobre larvas de lepidópteros en caña de azúcar en Colombia. En las zonas paneleras del país se han identificado parasitoides de los géneros Billaea, Genea y Alabagrus y la especie C. flavipes sobre barrenadores del género Diatraea, estos controladores pueden alcanzar un parasitismo del $38 \%$ en la Hoya del río Suárez (Sandoval et al. 2015; Sarmiento et al. 2017).

El control biológico natural que están ejerciendo los parasitoides mencionados muestra su importancia como reguladores de los barrenadores e indica la necesidad de caracterizar la diversidad de enemigos naturales en el cultivo de caña de azúcar para la producción de panela. Este conocimiento es la base para investigar su potencial en el manejo de las plagas del cultivo. En consecuencia, el objetivo de esta investigación fue proveer registros de los parasitoides Braconidae (Hymenoptera) y Tachinidae (Diptera) de los barrenadores, Crambidae y Coleophoridae (Lepidoptera) de caña azúcar para la industria panelera de la Hoya del río Suárez a partir de un muestreo intensivo específico.

\section{Materiales y métodos}

Los parasitoides se obtuvieron de larvas barrenadoras de tallo de cultivos de caña de azúcar, recolectadas durante el desarrollo del proyecto Fluctuación poblacional de Diatraea spp. y sus parasitoides en la Hoya del río Suárez realizado por la Corporación Colombiana de Investigación Agropecuaria-Agrosavia entre febrero de 2015 y febrero de 2017 en lotes de nueve fincas productoras de caña para panela ubicadas en los municipios de Chitaraque, Santana y Moniquirá del departamento de Boyacá y Ocamonte, Páramo y Valle de San José del departamento de Santander, Colombia. La variedad de caña predominante en el área es RD 75-11 excepto en Moniquirá, en donde se destaca una variedad de identidad desconocida a la que los agricultores han denominado "caña buena". La recolección de las larvas de las especies $D$. saccharalis, D. busckella, Diatraea pos. rosa, E. insuastii y de Blastobasis sp., se realizó amparada con permiso de la Agencia Nacional de Licencias Ambientales colombiana ANLA (Resolución 1466 del 03 de diciembre de 2014). Se revisaron 616 especímenes parasitoides a partir de las 4108 larvas recolectadas en cultivos de caña menores a cinco meses de edad con el síntoma de corazón muerto, reconocido por el marchitamiento de la hoja que conforma el cogollo y la yema terminal, enseguida, se realizaron cortes en los tallos de caña y se extrajeron las larvas. El esfuerzo de muestreo mensual fue de 2 horas/hombre/lote por finca, las larvas se colocaron en recipientes plásticos y se trasladaron al laboratorio de entomología del Centro de Investigación Tibaitatá
Agrosavia, en donde se individualizaron en dieta artificial de Hensley y Hammond (1968) en condiciones de $27^{\circ} \mathrm{C} \pm 2$; $50 \% \pm 10$ H.R. y fotoperiodo de 12 horas y se realizó un seguimiento hasta obtener la polilla adulta o un parasitoide. Los especímenes se preservaron en etanol $75 \%$ debidamente etiquetados y algunos fueron montados en alfiler según proceso estándar en entomología. Para la determinación de los ejemplares de Braconidae se usaron las claves de Wharton et al. (1993), Leathers y Sharkey (2003) y Muirhead et al. (2008); para los especímenes de Tachinidae se siguieron las claves de Brown et al. (2010), Guimaraes (1977), Nunez y Couri (2011) y O’Hara (1982). Los especímenes fueron depositados en la Colección Taxonómica Nacional Luis María Murillo (CTNI), AGROSAVIA, Centro de Investigación Tibaitatá.

\section{Resultados}

Se encontraron seis especies de Braconidae, cinco correspondieron al género Alabagrus, a saber: A. albispina (Cameron, 1887), A. imitatus (Cresson, 1873), A. parvifaciatus (Cameron, 1911), A. roibasi Sharkey, 1988, A. stigma y la especie Cotesia flavipes (Fig. 1). De Tachinidae se encontraron cuatro taxones: Billaea claripalpis, Genea jaynesi, Leskia sp. y Phytomyptera sp. (Fig. 2) (Tabla 1). Se preservó un espécimen de cada especie del género Alabagrus, dos muestras de eventos de parasitismo de C. flavipes, tres especímenes de B. claripalpis, dos de G. jaynesi, uno de Leskia sp. y uno de Phytomyptera $\mathrm{sp}$.

\section{Alabagrus albispina (Cameron, 1887)}

Material examinado. Alabagrus albispina. $1 \hat{\sigma}$. COLOMBIA. Santander, Ocamonte, $06^{\circ} 21^{\prime} 52^{\prime \prime} \mathrm{N}, 7^{\circ} 08^{\prime} 21^{\prime \prime} \mathrm{O}, 1672$ $\mathrm{m}$, en larva de Diatraea sp. (Crambidae). En cultivo de Saccharum officinarum (Poaceae)-caña de azúcar para panela, 09-jul-2015. J, Gómez; J. Jiménez. [CTNI].

\section{Alabagrus imitatus (Cresson, 1873)}

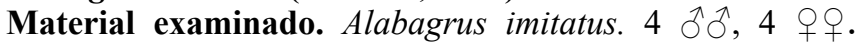
COLOMBIA. Boyacá, Santana, 0601'46"N, 73²9'21"O, $1738 \mathrm{~m}$, en larvas de Diatraea sp. (Crambidae). En cultivo de Saccharum officinarum (Poaceae)-caña de azúcar para panela, oct-2016. J, Gómez; J. Jiménez. [CTNI].

\section{Alabagrus parvifaciatus (Cameron, 1911)}

Material examinado. Alabagrus parvifaciatus. 1 q. COLOMBIA. Boyacá, Moniquirá, 055 $56^{\prime} 11^{\prime \prime} \mathrm{N}, 73^{\circ} 31^{\prime} 50^{\prime \prime} \mathrm{O}$, $1650 \mathrm{~m}$, en larva de Diatraea sp. (Crambidae). En cultivo de Saccharum officinarum (Poaceae)-caña de azúcar para panela, sep-2015. J, Gómez; J. Jiménez. [CTNI].

\section{Alabagrus roibasi Sharkey, 1988}

Material examinado. Alabagrus roibasi. 1 ๙ . COLOMBIA. Boyacá, Moniquirá, $05^{\circ} 56^{\prime} 11^{\prime \prime} \mathrm{N}, 73^{\circ} 31^{\prime} 50^{\prime \prime} \mathrm{O}, 1650 \mathrm{~m}$, en larva de Diatraea sp. (Crambidae). En cultivo de Saccharum officinarum (Poaceae)-caña de azúcar para panela, 27-may2016. J, Gómez; J. Jiménez. [CTNI].

\section{Alabagrus stigma (Brullé, 1846)}

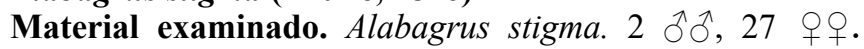
COLOMBIA. Boyacá, Moniquirá, $05^{\circ} 56^{\prime} 11^{\prime \prime} \mathrm{N}, 73^{\circ} 31^{\prime} 50^{\prime \prime} \mathrm{O}$, $1650 \mathrm{~m}$, en larvas de Diatraea sp. (Crambidae). En cultivo de Saccharum officinarum (Poaceae)-caña de azúcar para panela, may-2015. J, Gómez; J. Jiménez. [CTNI]. 


\section{Cotesia flavipes Cameron, 1891}

Material examinado. Cotesia flavipes. 150 especímenes (sin sexar). COLOMBIA. Santander, Páramo, $06^{\circ} 26^{\prime} 30^{\prime \prime} \mathrm{N}$,

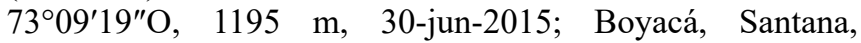
$06^{\circ} 01^{\prime} 46^{\prime \prime} \mathrm{N}, 7^{\circ} 29^{\prime} 21^{\prime \prime} \mathrm{O}, 1738 \mathrm{~m}, 13$-ago-2015. En larvas de Diatraea sp. (Crambidae), en cultivo de Saccharum officinarum (Poaceae)-caña de azúcar para panela. J, Gómez; J. Jiménez. [CTNI].

\section{Billaea claripalpis (Wulp, 1896)}

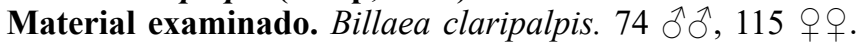
COLOMBIA. Boyacá, Moniquirá, $05^{\circ} 56^{\prime} 55^{\prime \prime} \mathrm{N}, 73^{\circ} 32^{\prime} 48^{\prime \prime} \mathrm{O}$, $1612 \mathrm{~m}, 20$-dic-2015; Moniquirá, $05^{\circ} 55^{\prime} 10^{\prime \prime} \mathrm{N}, 73^{\circ} 32^{\prime} 59^{\prime \prime} \mathrm{O}$, 1960 m, nov-2016; Santana, 06 $01^{\prime} 46^{\prime \prime} \mathrm{N} 73^{\circ} 29^{\prime} 21^{\prime \prime} \mathrm{O}, 1738$ m, 1-jul-2015. En larvas de Diatraea sp. (Crambidae), en cultivo de Saccharum officinarum (Poaceae)-caña de azúcar para panela. J, Gómez; J. Jiménez. [CTNI].

\section{Genea jaynesi (Aldrich, 1932)}

Material examinado. Genea jaynesi. 113 $\precsim \widehat{\jmath}, 117$ 우우. COLOMBIA. Santander, Páramo, 06 $26^{\prime} 30^{\prime \prime} \mathrm{N} 73^{\circ} 09^{\prime} 19^{\prime \prime} \mathrm{O}, 1195$ $\mathrm{m}$, dic-2015; Ocamonte, $06^{\circ} 21^{\prime} 52^{\prime \prime} \mathrm{N}, 73^{\circ} 08^{\prime} 21^{\prime \prime} \mathrm{O}, 1672 \mathrm{~m}$, dic-2015. En larva de Diatraea sp. (Crambidae). En cultivo de Saccharum officinarum (Poaceae)-caña de azúcar para panela. J, Gómez; J. Jiménez. [CTNI].

\section{Leskia sp.}

Material examinado. Leskia sp. 1 espécimen sin sexar. COLOMBIA. Santander, Ocamonte, $06^{\circ} 21^{\prime} 52^{\prime \prime} \mathrm{N}, 7^{\circ} 08^{\prime} 21^{\prime \prime} \mathrm{O}$, 1672 m, en larva de Diatraea sp. (Crambidae). En cultivo de Saccharum officinarum (Poaceae)-caña de azúcar para panela, ene-2016. J, Gómez; J. Jiménez. [CTNI].

\section{Phytomyptera sp.}

Material examinado. Phytomyptera sp., 1 espécimen sin sexar. COLOMBIA. Boyacá, Santana, 06 $02^{\prime} 06^{\prime \prime N}$, 7329'51"O, $1593 \mathrm{~m}$, en larva de Blastobasis sp. (Coleophoridae). En cultivo de Saccharum officinarum (Poaceae)-caña de azúcar para panela, abril-2016. J, Gómez; J. Jiménez. [CTNI].

\section{Discusión}

Los géneros Cotesia Cameron, 1891, y Alabagrus Enderlein, 1920, fueron registrados en Colombia en 1970 y 2001, respectivamente. Cotesia flavipes fue introducida al país mediante liberaciones masivas en el Valle del río Cauca y en el departamento de Santander y se registró en la región andina y oriental como controlador biológico de especies lepidópteros (Vargas et al. 2015). Por su parte, Alabagrus se registró en las regiones naturales del Amazonas y Caribe parasitando lepidópteros de la familia Pyralidae (Campos 2001).

Actualmente, C. flavipes se produce masivamente para el control de barrenadores. Algunos de sus hospedantes son $D$. saccharalis y otras especies del género Diatraea en cultivos de caña de azúcar del continente americano y El Caribe; en Brasil es el principal controlador biológico utilizado para regular las poblaciones de barrenadores (Badilla 2002; White et al. 2004; Parra et al. 2010). En Colombia, las liberaciones masivas de C. flavipes realizadas entre 1975 y 1982 conllevaron al establecimiento del parasitoide en los departamentos de Boyacá y Norte de Santander y solamente hasta comienzos de la década del 2010 se logró su establecimiento en la región del Valle del río Cauca gracias a las liberaciones hechas para controlar la aparición de D. tabernella y D. busckella (Vargas et al. 2013, 2015; Aya et al. 2017). Arboleda y Vargas (2019)

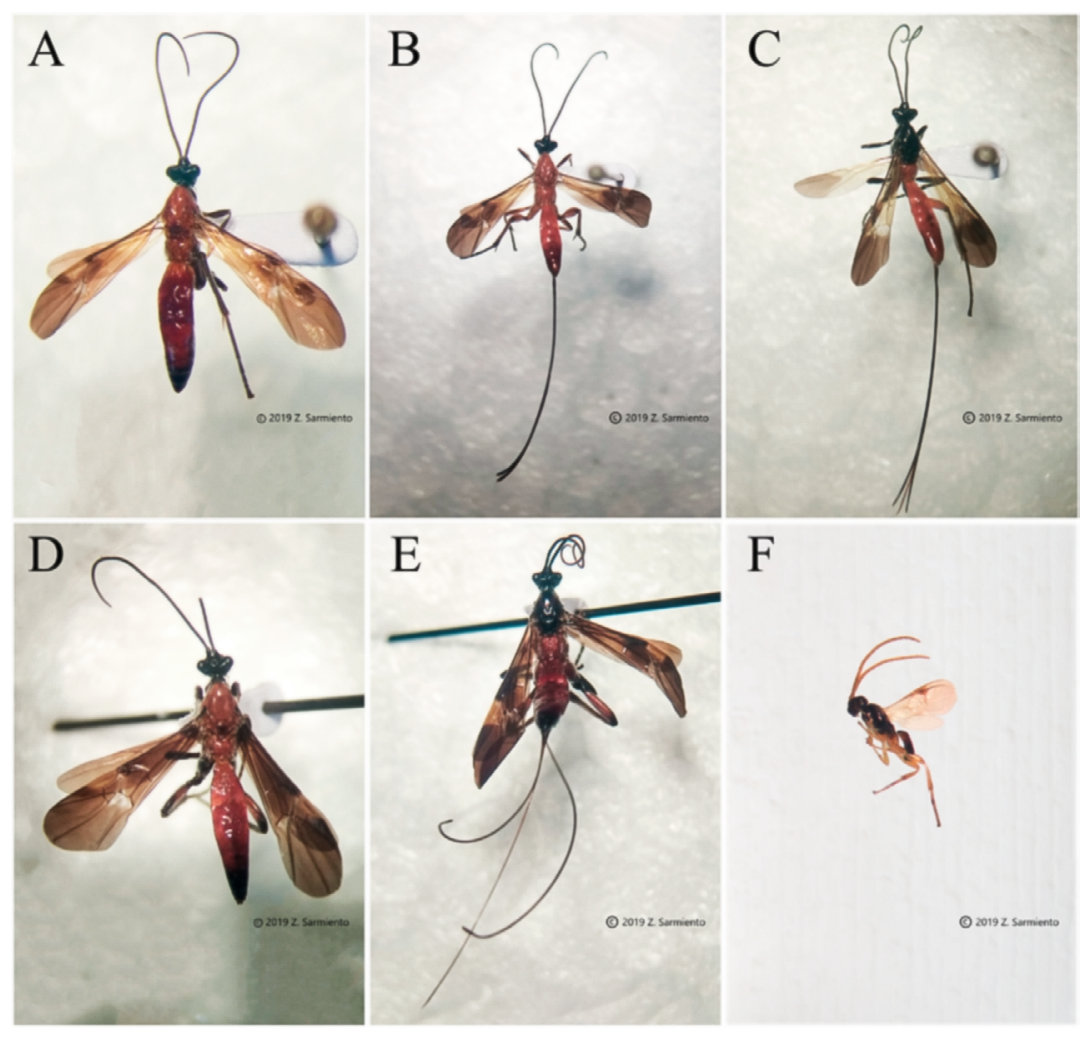

Figura 1. Bracónidos registrados en las larvas de barrenadores. A. Alabagrus albispina. B. A. imitatus. C. A. parvifaciatus. D. A. roibasi. E. A. stigma y F. Cotesia flavipes. 

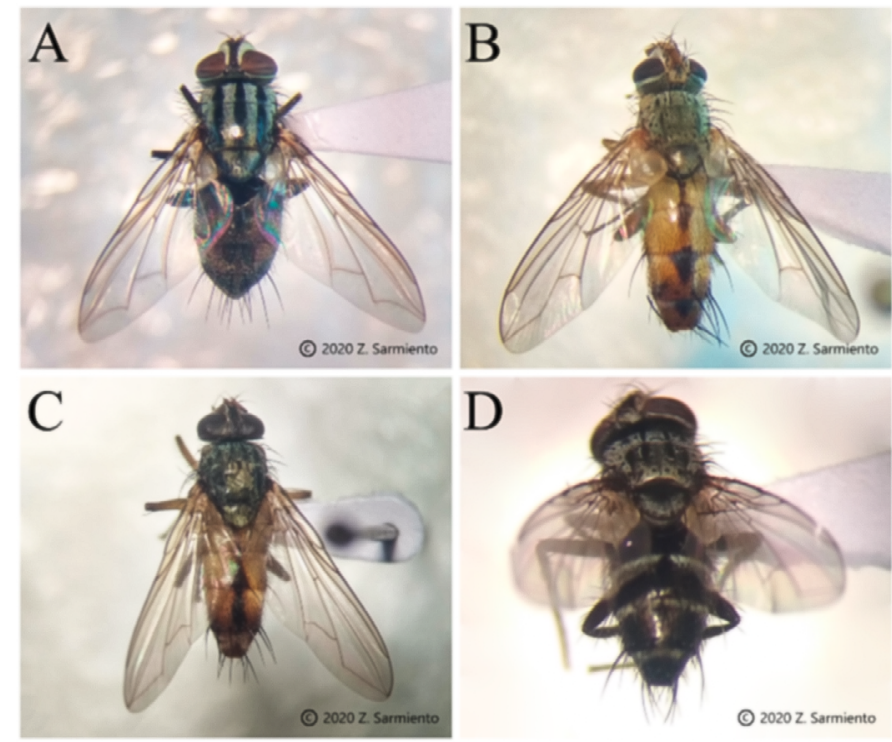

Figura 2. Taquínidos registrados en las larvas de barrenadores. A. Billaea claripalpis. B. Genea jaynesi. C. Leskia sp. D. Phytomyptera sp.

Tabla 1. Parasitoides y sus hospedantes en caña de azúcar para panela en los departamentos de Boyacá y Santander, Colombia.

\begin{tabular}{|c|c|c|c|c|c|c|c|c|}
\hline Especie de parasitoide & Hospedantes & Departamento & Municipio & Latitud (N) & Longitud (O) & $\begin{array}{c}\text { Altitud } \\
\text { (m.s.n.m) }\end{array}$ & Colectores & Repositorio \\
\hline Alabagrus albispina $(\mathrm{n}=1)$ & Diatraea sp. & Santander & Ocamonte & $06^{\circ} 21^{\prime} 52^{\prime \prime}$ & $73^{\circ} 08^{\prime} 21^{\prime \prime}$ & 1672 & $\begin{array}{l}\text { J. Gómez; } \\
\text { J. Jiménez }\end{array}$ & CTNI \\
\hline Alabagrus imitatus $(\mathrm{n}=8)$ & $\begin{array}{l}\text { Diatraea } \mathrm{sp} . \\
\text { E. insuastii }\end{array}$ & $\begin{array}{l}\text { Santander } \\
\text { Boyacá }\end{array}$ & $\begin{array}{l}\text { Páramo } \\
\text { Chitaraque } \\
\text { Santana } \\
\text { Moniquirá }\end{array}$ & $\begin{array}{l}06^{\circ} 26^{\prime} 30^{\prime \prime} \\
05^{\circ} 59^{\prime} 40^{\prime \prime} \\
06^{\circ} 01^{\prime} 46^{\prime \prime} \\
05^{\circ} 56^{\prime} 11^{\prime \prime} \\
05^{\circ} 56^{\prime} 55^{\prime \prime}\end{array}$ & $\begin{array}{l}73^{\circ} 09^{\prime} 19^{\prime \prime} \\
73^{\circ} 27^{\prime} 47^{\prime \prime} \\
73^{\circ} 29^{\prime} 21^{\prime \prime} \\
73^{\circ} 31^{\prime} 50^{\prime \prime} \\
73^{\circ} 32^{\prime} 48^{\prime \prime}\end{array}$ & $\begin{array}{l}1195 \\
1723 \\
1738 \\
1650 \\
1612\end{array}$ & $\begin{array}{l}\text { J. Gómez; } \\
\text { J. Jiménez }\end{array}$ & CTNI \\
\hline Alabagrus parvifaciatus $(\mathrm{n}=1)$ & Diatraea sp. & Boyacá & Moniquirá & $05^{\circ} 56^{\prime} 11^{\prime \prime}$ & $73^{\circ} 31^{\prime} 50^{\prime \prime}$ & 1650 & $\begin{array}{l}\text { J. Gómez; } \\
\text { J. Jiménez }\end{array}$ & CTNI \\
\hline Alabagrus roibasi $(\mathrm{n}=1)$ & Diatraea sp. & Boyacá & Moniquirá & $05^{\circ} 56^{\prime} 11^{\prime \prime}$ & $73^{\circ} 31^{\prime} 50^{\prime \prime}$ & 1650 & $\begin{array}{l}\text { J. Gómez; } \\
\text { J. Jiménez }\end{array}$ & CTNI \\
\hline Alabagrus stigma $(\mathrm{n}=29)$ & $\begin{array}{l}\text { Diatraea } \mathrm{sp} . \\
\text { E. insuastii }\end{array}$ & $\begin{array}{l}\text { Santander } \\
\text { Boyacá }\end{array}$ & $\begin{array}{l}\text { Páramo } \\
\text { Moniquirá }\end{array}$ & $\begin{array}{l}06^{\circ} 26^{\prime} 30^{\prime \prime} \\
05^{\circ} 56^{\prime} 11^{\prime \prime} \\
05^{\circ} 56^{\prime} 55^{\prime \prime} \\
05^{\circ} 55^{\prime} 10^{\prime \prime}\end{array}$ & $\begin{array}{l}73^{\circ} 09^{\prime} 19^{\prime \prime} \\
73^{\circ} 31^{\prime} 50^{\prime \prime} \\
73^{\circ} 32^{\prime} 48^{\prime \prime} \\
73^{\circ} 32^{\prime} 59^{\prime \prime}\end{array}$ & $\begin{array}{l}1195 \\
1650 \\
1612 \\
1960\end{array}$ & $\begin{array}{l}\text { J. Gómez; } \\
\text { J. Jiménez }\end{array}$ & CTNI \\
\hline Cotesia flavipes $(\mathrm{n}=150)$ & $\begin{array}{l}\text { Diatraea } \mathrm{sp} . \\
\text { E. insuastii }\end{array}$ & $\begin{array}{l}\text { Santander } \\
\text { Boyacá }\end{array}$ & $\begin{array}{l}\text { Ocamonte } \\
\text { Páramo } \\
\text { Valle de San José } \\
\text { Santana } \\
\text { Moniquirá }\end{array}$ & $\begin{array}{l}06^{\circ} 21^{\prime} 52^{\prime \prime} \\
06^{\circ} 26^{\prime} 30^{\prime \prime} \\
06^{\circ} 28^{\prime} 42^{\prime \prime} \\
06^{\circ} 02^{\prime} 06^{\prime \prime} \\
06^{\circ} 01^{\prime} 46^{\prime \prime} \\
05^{\circ} 56^{\prime} 11^{\prime \prime} \\
05^{\circ} 56^{\prime} 55^{\prime \prime} \\
05^{\circ} 55^{\prime} 10^{\prime \prime}\end{array}$ & $\begin{array}{l}73^{\circ} 08^{\prime} 21^{\prime \prime} \\
73^{\circ} 09^{\prime} 19^{\prime \prime} \\
73^{\circ} 04^{\prime} 55^{\prime \prime} \\
73^{\circ} 29^{\prime} 51^{\prime \prime} \\
73^{\circ} 29^{\prime} 21^{\prime \prime} \\
73^{\circ} 31^{\prime} 50^{\prime \prime} \\
73^{\circ} 32^{\prime} 48^{\prime \prime} \\
73^{\circ} 32^{\prime} 59^{\prime \prime}\end{array}$ & $\begin{array}{l}1672 \\
1195 \\
1308 \\
1593 \\
1738 \\
1650 \\
1612 \\
1960\end{array}$ & $\begin{array}{l}\text { J. Gómez; } \\
\text { J. Jiménez }\end{array}$ & CTNI \\
\hline Billaea claripalpis $(\mathrm{n}=189)$ & $\begin{array}{l}\text { Diatraea } \mathrm{sp} . \\
\text { E. insuastii }\end{array}$ & $\begin{array}{l}\text { Santander } \\
\text { Boyacá }\end{array}$ & $\begin{array}{l}\text { Ocamonte } \\
\text { Páramo } \\
\text { Valle de San José } \\
\text { Chitaraque } \\
\text { Santana } \\
\text { Moniquirá }\end{array}$ & $\begin{array}{l}06^{\circ} 21^{\prime} 52^{\prime \prime} \\
06^{\circ} 26^{\prime} 30^{\prime \prime} \\
06^{\circ} 28^{\prime} 42^{\prime \prime} \\
05^{\circ} 59^{\prime} 40^{\prime \prime} \\
06^{\circ} 02^{\prime} 06^{\prime \prime} \\
06^{\circ} 01^{\prime} 46^{\prime \prime} \\
05^{\circ} 56^{\prime} 11^{\prime \prime} \\
05^{\circ} 56^{\prime} 55^{\prime \prime} \\
05^{\circ} 55^{\prime} 10^{\prime \prime}\end{array}$ & $\begin{array}{l}73^{\circ} 08^{\prime} 21^{\prime \prime} \\
73^{\circ} 09^{\prime} 19^{\prime \prime} \\
73^{\circ} 04^{\prime} 55^{\prime \prime} \\
73^{\circ} 27^{\prime} 47^{\prime \prime} \\
73^{\circ} 29^{\prime} 51^{\prime \prime} \\
73^{\circ} 29^{\prime} 21^{\prime \prime} \\
73^{\circ} 31^{\prime} 50^{\prime \prime} \\
73^{\circ} 32^{\prime} 48^{\prime \prime} \\
73^{\circ} 32^{\prime} 59^{\prime \prime}\end{array}$ & $\begin{array}{l}1672 \\
1195 \\
1308 \\
1723 \\
1593 \\
1738 \\
1650 \\
1612 \\
1960 \\
\end{array}$ & $\begin{array}{l}\text { J. Gómez; } \\
\text { J. Jiménez }\end{array}$ & CTNI \\
\hline Genea jaynesi $(\mathrm{n}=230)$ & $\begin{array}{l}\text { Diatraea } \mathrm{sp} . \\
\text { E. insuastii }\end{array}$ & Boyacá & $\begin{array}{l}\text { Ocamonte } \\
\text { Páramo } \\
\text { Valle de San José } \\
\text { Chitaraque } \\
\text { Santana }\end{array}$ & $\begin{array}{l}06^{\circ} 21^{\prime} 52^{\prime \prime} \\
06^{\circ} 26^{\prime} 30^{\prime \prime} \\
06^{\circ} 28^{\prime} 42^{\prime \prime} \\
05^{\circ} 59^{\prime} 40^{\prime \prime} \\
06^{\circ} 02^{\prime} 06^{\prime \prime} \\
06^{\circ} 01^{\prime} 46^{\prime \prime}\end{array}$ & $\begin{array}{l}73^{\circ} 08^{\prime} 21^{\prime \prime} \\
73^{\circ} 09^{\prime} 19^{\prime \prime} \\
73^{\circ} 04^{\prime} 55^{\prime \prime} \\
73^{\circ} 27^{\prime} 47^{\prime \prime} \\
73^{\circ} 29^{\prime} 51^{\prime \prime} \\
73^{\circ} 29^{\prime} 21^{\prime \prime}\end{array}$ & $\begin{array}{l}1672 \\
1195 \\
1308 \\
1723 \\
1593 \\
1738\end{array}$ & $\begin{array}{l}\text { J. Gómez; } \\
\text { J. Jiménez }\end{array}$ & CTNI \\
\hline
\end{tabular}




\begin{tabular}{|c|c|c|c|c|c|c|c|c|}
\hline Especie de parasitoide & Hospedantes & Departamento & Municipio & Latitud (N) & Longitud (O) & $\begin{array}{c}\text { Altitud } \\
\text { (m.s.n.m) }\end{array}$ & Colectores & Repositorio \\
\hline Leskia $\mathrm{sp} .(\mathrm{n}=1)$ & Diatraea sp. & Santander & Ocamonte & $06^{\circ} 21^{\prime} 52^{\prime \prime}$ & $73^{\circ} 08^{\prime} 21^{\prime \prime}$ & 1672 & $\begin{array}{l}\text { J. Gómez; } \\
\text { J. Jiménez }\end{array}$ & CTNI \\
\hline Phytomyptera sp. $(\mathrm{n}=6)$ & Blastobasis sp. & $\begin{array}{l}\text { Santander } \\
\text { Boyacá }\end{array}$ & $\begin{array}{l}\text { Valle de San José } \\
\text { Chitaraque } \\
\text { Santana } \\
\text { Moniquirá }\end{array}$ & $\begin{array}{l}06^{\circ} 28^{\prime} 42^{\prime \prime} \\
05^{\circ} 59^{\prime} 40^{\prime \prime} \\
06^{\circ} 02^{\prime} 06^{\prime \prime} \\
05^{\circ} 55^{\prime} 10^{\prime \prime}\end{array}$ & $\begin{array}{l}73^{\circ} 04^{\prime} 55^{\prime \prime} \\
73^{\circ} 27^{\prime} 47^{\prime \prime} \\
73^{\circ} 29^{\prime} 51^{\prime \prime} \\
73^{\circ} 32^{\prime} 59^{\prime \prime}\end{array}$ & $\begin{array}{l}1308 \\
1723 \\
1593 \\
1960\end{array}$ & $\begin{array}{l}\text { J. Gómez; } \\
\text { J. Jiménez }\end{array}$ & CTNI \\
\hline
\end{tabular}

reportaron el potencial de $C$. flavipes como enemigo natural de $D$. tabernella. Recientemente, se reveló que además de tener como hospedantes a estas dos especies de Diatraea, en el Valle del río Cauca hay otras dos especies alternativas $D$. saccharalis y $D$. indigenella siendo la segunda ligeramente más adecuada que la primera (Londoño-Sánchez et al. 2020). En el presente trabajo, $C$. flavipes se registra parasitando a $E$. insuastii y Diatraea spp. en Santander y Boyacá. En tanto no hay liberaciones recientes de $C$. flavipes en estos dos departamentos, los registros muestran un proceso de establecimiento exitoso en la zona.

Los ejemplares de A. albispina, A. parvifaciatus, A. roibasi y tres individuos de $A$. imitatus presentaron carenas del primer tergo metasomal sin fusión medial, variación poco frecuente en el género según Leathers y Sharkey (2003). Además, la coloración de la metapleura, pronoto y mesonoto de los ejemplares de $A$. albispina presentó una variación, ante esto, se conoce que en los agatidinos pueden variar los caracteres de coloración (Sharkey 1988).

Alabagrus albispina se encuentra en América Central, El Caribe y América del Sur y sus hospedantes son enrolladores de hojas de la familia Crambidae (Leathers y Sharkey 2003); esta especie se reporta por primera vez en caña de azúcar para la producción de panela parasitando a taxones del género Diatraea, en el departamento de Santander, Colombia. Alabagrus parvifaciatus se distribuye desde el sur de Honduras hasta Ecuador y del este de Ecuador hasta Brasil, se conocen como hospedantes a especies de Diatraea (Sharkey 1998). En este estudio, A. albispina se reporta en Boyacá. Alabagrus roibasi está extendida desde el sur de México al norte de Costa Rica; sus hospedantes conocidos son crámbidos enrolladores de hojas y un herbívoro de solanáceas del género Herpetogramma (Pyralidae) (Leathers y Sharkey 2003; Gentry 2003). En este trabajo, A. roibasi se reporta por primera vez sobre larvas de Diatraea spp. y su distribución se extiende hasta Boyacá, Colombia.

Alabagrus imitatus (Cresson, 1873) se distribuye en el sureste de los Estados Unidos y México y parasita especies de Diatraea, tales como D. mitteri (Leathers y Sharkey 2003; Solis et al. 2015). En este estudio se reporta por primera vez a $A$. imitatus sobre E. insuastii en Colombia en los departamentos de Santander y Boyacá, además de parasitar larvas del género Diatraea. Varias especies del género Diatraea conviven en la zona de estudio por lo que no fue posible determinar cuál o cuáles especies parasita $A$. imitatus, pero se tratarán de registros nuevos para la especie. Otra especie encontrada fue $A$. stigma, esta avispa está extendida desde el sur de La Florida hasta Uruguay y norte de Argentina; se conocen como hospedantes a especies de Diatraea en arroz, caña de azúcar y maíz (Sharkey 1988; Leathers y Sharkey 2003); en el sur de Texas parasita a D. saccharalis y a Eoreuma loftini (Dyar, 1917) (Hall et al. 2007). En el presente estudio, A. stigma se reporta en larvas de Diatraea spp. y de E. insuastii, en Santander y en Boyacá.
El taquínido B. claripalpis está distribuido en toda la región neotropical excepto Chile y Sur de Argentina (Guimaraes 1977). En Brasil B. claripalpis se ha empleado en el control de D. saccharalis y en Colombia se ha producido masivamente para su liberación en cultivos de caña de azúcar afectados por especies de Diatraea (Vargas et al. 2015; Borges et al. 2019). Aya et al. (2019) al evaluar la idoneidad de cuatro especies de Diatraea como hospedantes de $B$. claripalpis encontraron un porcentaje de parasitismo menor en $D$. tabernella y $D$. busckella que en $D$. saccharalis y D. indigenella sugiriendo una posible resistencia por parte de las dos primeras especies. Billaea claripalpis se reporta por primera vez en Santander y Boyacá, con un alto número de especímenes $(\mathrm{n}=189)$ parasitando a Diatraea spp. y E. insuastii. Otra especie de taquínido encontrada fue $G$. jaynesi, la cual está distribuida en Argentina, Brasil, Colombia y Venezuela; D. impersonatella y D. saccharalis se le conocen como hospedantes (Nunez y Couri 2011). En Colombia, G. jaynesi se encuentra establecida en condiciones naturales en el Valle del Cauca parasitando especies de Diatraea y no ha sido posible su cría en laboratorio (Vargas et al. 2015). En este estudio G. jaynesi fue el parasitoide con el mayor número de individuos $(\mathrm{n}=230)$ y se reporta sobre Diatraea spp. y E. insuastii en Santander y en Boyacá.

Por último, se registró un taquínido perteneciente al género Leskia Robineau-Desvoidy, 1830. Este género se encuentra distribuido desde la región paleártica, desde el norte de México hasta la región neotropical (Brown et al. 2010); lepidópteros de Pyralidae y Sesiidae se conocen como hospedantes (Arnaud 1978). El género Leskia se registra por primera vez sobre Diatraea en caña de azúcar para la producción de panela en Santander. El género Phytomyptera Rondani, 1844, tiene especies registradas al norte de México y en la región neotropical (Brown et al. 2010); parasita varias especies de lepidópteros en Norteamérica y a $B$. graminea en caña de azúcar en Colombia (Bustillo 2011; Arnaud 1978). En el presente trabajo, el género Leskia se reporta en los departamentos de Santander y Boyacá parasitando una especie del género Blastobasis.

El conocimiento de las especies que atacan naturalmente las plagas de cultivos es fase fundamental para el desarrollo de aplicaciones de control biológico. Bustillo (2011) registró doce parasitoides controlando larvas de lepidópteros en caña de azúcar en Colombia. En el presente trabajo se registran diez especies establecidas en caña de azúcar para panela, de las cuales cinco se amplían para el país y para las especies de barrenadores lepidópteros en caña de azúcar, tres de ellas $A$. albispina, A. roibasi y Leskia sp. son registros nuevos para los herbívoros del cultivo, lo que amplía el número de parasitoides de los barrenadores de caña, tan importantes en la producción de panela y, además, sugiere que aún hay especies por caracterizar como enemigos naturales en las zonas paneleras del país. 
En esta región, organizaciones de productores como FEDEPANELA y gubernamentales como el ICA suministran ocasionalmente los parasitoides T. exiguum y L. minense a los productores para realizar liberaciones siguiendo las recomendaciones de Tarazona (2011) y el ICA (2017). Sin embargo, no se encontraron individuos parasitados por L. minense, lo que parece indicar que, hasta el momento, no se ha establecido en la región.

Teniendo en cuenta que las especies C. flavipes, B. claripalpis y $G$. jaynesi fueron las más frecuentes, se debería apalancar su conservación en los agroecosistemas de caña de azúcar. Una de las estrategias promisorias para potenciar el parasitismo natural en agroecosistemas de caña de azúcar es la sugerida por el ICA (2017) y por Vargas et al. (2006), que consiste en el mantenimiento de franjas de plantas con flores nectaríferas cercanas a los lotes del cultivo para favorecer la abundancia de estos enemigos naturales.

\section{Agradecimientos}

Los autores agradecen a la Corporación Colombiana de Investigación Agropecuaria (Agrosavia) por la financiación de la investigación a través del proyecto "Estrategias de manejo integrado de plagas y enfermedades asociadas a cultivos de caña de azúcar en regiones productoras de panela en Colombia". A Pablo Osorio, Orlando Insuasty, Yuly Sandoval, Jesús Gómez, Javier Jiménez, Sandra Romero, Liliana Cely y John Gallo, de Agrosavia. A Catalina Romero, Lina Lozano y Andrea Román del grupo de Sistemática y Biología Comparada de insectos de la Universidad Nacional de Colombia. A los pares evaluadores y al equipo editorial de la revista.

\section{Literatura citada}

ARBOLEDA, B.; VARGAS, G. 2019. Efficacy of Cotesia flavipes (Hymenoptera: Braconidae) in reducing Diatraea tabernella (Lepidoptera: Crambidae) injury in sugarcane. Florida Entomologist 102 (3): 520-525. https://doi. org/10.1653/024.102.0307

ARNAUD, P. H.; J. R. 1978. A host-parasite catalog of North American Tachinidae (Diptera). United States Department of Agriculture. Miscellaneous Publication 1319: 1-860.

AYA, V. M; ECHEVERRI-RUBIANO, C.; BARRERA, G. P.; VARGAS, G. 2017. Cotesia flavipes (Hymenoptera: Braconidae) as a biological control agent of sugarcane stem borers in Colombia's Cauca River Valley. Florida Entomologist 100 (4): 826-830. https://doi.org/10.1653/024.100.0426

AYA, V. M.; MONTOYA-LERMA, J.; ECHEVERRI-RUBIANO, C.; MICHAUD, J. P.; VARGAS, G. 2019. Host resistance to two parasitoids (Diptera: Tachinidae) helps explain a regional outbreak of novel Diatraea spp. stem borers (Lepidoptera: Crambidae) in Colombia sugarcane. Biological Control 129 (1): 18-23. https://doi.org/10.1016/j.biocontrol.2018.11.009

BADILLA, F. 2002. Un programa exitoso de control biológico de insectos plaga de la caña de azúcar en Costa Rica. Manejo Integrado de Plagas y Agroecología 64: 77-87. http://repositorio. bibliotecaorton.catie.ac.cr/bitstream/handle/11554/6868/ A2050e.pdf?sequence $=1$ \&isAllowed $=y$

BORGES FILHO, R.; DA C. STURZA, V. S.; BERNARDI, D.; DA CUNHA, U. S.; PINTO A. S.; DOS ANGOS E SILVA, S. D.; NAVA, D. E. 2019. Population dynamics of pest and natural enemies on sugar cane grown in a subtropical region of Brazil. Florida Entomologist 102 (3): 526-530. https://doi. org/10.1653/024.102.0313

BROWN, B.V.; BORKENT, A.; CUMMING, J.M.; WOOD, D. M.; WOODLEY, N.E.; ZUMBADO, M. A. 2010. Manual of Central
American Diptera. Volumen 2. NRC Research Press, Ottawa, Ontario Canadá, 728 p.

BUSTILLO, A. E. 2011. Parasitoides, depredadores y entomopatógenos que afectan las plagas de la caña de azúcar en Colombia. Centro de Investigación de la caña de azúcar en Colombia. Cenicaña. Cali, Colombia, 25 p. https://www. cenicana.org/pdf_privado/no_clasificacion/6481.pdf

CAMPOS, D. 2001. Lista de los géneros de avispas parasitoides Braconidae (Hymenoptera: Ichneumonoidea) de la región neotropical. Biota Colombiana 2 (3): 193-232. http://repository. humboldt.org.co/bitstream/handle/20.500.11761/32724/100100-1-PB.pdf? sequence $=1 \&$ isAllowed $=y$

GENTRY, G. 2003. Multiple parasitoid visitors to the extrafloral nectaries of Solanum adherens. Is $S$. adherens an insectary plant?. Basic and Applied Ecology 4 (5): 405411. https://doi. org/10.1078/1439-1791-00189

GÓMEZ, L. A.; DÍAZ A. E.; LASTRA L. A. 1996. Survey of the Trichogramma species associated with sugarcane in Colombia. Revista Colombiana de Entomología 22: 1-5.

GUIMARAES, J. H. 1977. A revision of the genus Paratheresia Towsend (Diptera: Tachinidae, Theresiini). Papéis Avulsos de Zoologia 30 (18): 267-288.

HALL, D. G.; NUESSLY, G. S.; GILBERT, R. A. 2007. The sugarcane borer in Florida. University of Florida, Gainesville, Florida, USA. 5 p. https://ufdc.ufl.edu/IR00003041/00001

ICA- Instituto Colombiano Agropecuario. 2017. Resolución $\mathrm{N}^{\circ} 00017848$ del 20 de diciembre de 2017. "Por medio de la cual se establecen medidas fitosanitarias en el cultivo de caña de azúcar (Saccharum spp. L.) en el territorio nacional para la vigilancia y control de las especies barrenadoras del tallo del género Diatraea Guilding". 10 p. https:// www.ica.gov.co/getattachment/5491c441-fc40-4a4c-800ee5efb6177b86/2017R17848.aspx

LEATHERS, J.; SHARKEY, M. 2003. Taxonomy and life history of Costa Rican Alabagrus (Hymenoptera: Braconidae), with a key to world species. Contributions in science. Natural History Museum of Los Angeles County 497: 1-78. https://www. biodiversitylibrary.org/page/52221858\#page/79/mode/1up

LEYTON-FLOR, S. A.; GORDILLO, M.; GONZALEZ, P. A.; OSPINA, J. A.; VARGAS, G. 2018. Distribución espaciotemporal de Diatraea spp. (Lepidoptera: Crambidae) en el Valle del río Cauca, Colombia. Revista Colombiana de Entomología 44 (2): 177-186. 10.25100/socolen. v44i2.7330

LONDOÑO-SÁNCHEZ, C.; MONTOYA-LERMA, J.; MICHAUD, J.P.; VARGAS, G. 2020. The gregarious parasitoid Cotesia flavipes displays a high level of preadaptation to a novel host, Diatraea indigenella. BioControl 65: 37-46. https://doi. org/10.1007/s10526-019-09980-y

MUIRHEAD, K.; AUSTIN, A.; SALLAM, M. 2008. The systematics and biology of Cotesia nonagriae (Olliff) stat. rev. (Hymenoptera: Braconidae: Microgastrinae), a newly recognized member of the Cotesia flavipes complex. Zootaxa 1846 (1): 35-46. https://www. biotaxa.org/Zootaxa/article/view/zootaxa.1846.1.3/46227

NUNEZ, E.; COURI, M. 2011. Revision of Neotropical Genea Rondani (Diptera: Tachinidae: Tachininae, Leskiini). Papéis Avulsos de Zoologia 51 (31): 481-497. https://www.scielo.br/j/ paz/a/sCdyqKgr7gNRNNCGN4B8VSt/?format=pdf\&lang=en

O'HARA, J. E. 1982. Classification, phylogeny and zoogeography of the North American species of Siphona Meigen (Diptera: Tachinidae). Quaestiones Entomologicae 18 (1982): 261-380. https://nature.berkeley.edu/ kipwill/QE\%20documents\%20 for $\% 20$ public/OHara\%201982\%20QEv18n1_4\%20 261_380\%20CC\%20released.pdf

PARRA, J. R. P.; BOTELHO, P. S. M.; PINTO, A. DE S. 2010. Biological control of pests as a key component for sustainable sugarcane production, pp 441- 450 In Blucher E. Sugarcane Bioethanol R\&D for Productivity and Sustainability. Sao Paulo, Brasil. http://dx.doi.org/10.5151/BlucherOA-SugarcaneSUGARCANEBIOETHANOL 41 
POSADA, L. M.; GARCÍA, F. M. 1976. Lista de depredadores, parásitos y patógenos de insectos registrados en Colombia. Instituto Colombiano Agropecuario ICA. Boletín Técnico $\mathrm{N}^{\circ} 41.90$ p. https://repository.agrosavia. co/bitstream/handle/20.500.12324/13998/23648_5442. pdf?sequence $=1 \&$ isAllowed $=y$

SANDOVAL-CÁCERES, Y.; OSORIO-MEJIA, P.; GÓMEZ, J.; BARRETO-TRIANA, N.; ESPINEL, C.; VILLAMIZAR, L. 2015. Distribución de especies y enemigos naturales de Diatraea spp. (Lepidoptera: Crambidae) en caña panelera Saccharum officinarum L. en Colombia. Memorias y resúmenes, $42^{\circ}$ Congreso Sociedad Colombiana de entomología SOCOLEN. Medellín, Colombia. p 691. http://www.socolen.org.co/images/ stories/pdf/42 Congreso.pdf

SANDOVAL-CÁCERES, Y.; OSORIO-MEJIA, P.; SARMIENTONAIZAQUE, Z.; BARRETO-TRIANA, N. 2016. Biología del complejo de barrenadores del género Diatraea spp. (Lepidoptera: Crambidae) en dieta artificial. Resúmenes, $43^{\circ}$ Congreso Sociedad Colombiana de Entomología- SOCOLEN. Manizales, Colombia. p 186. http://www.socolen.org.co/publicaciones/ resumenes-43-congreso-socolen-2

SARMIENTO-NAIZAQUE, Z., OSORIO-MEJÍA, P., SANDOVALCÁCERES, Y., INSUASTY, O.; ROMERO, Y., BARRETOTRIANA, N. 2017. Parasitoides de Diatraea spp. (Lepidoptera: Crambidae) en caña para panela en Boyacá y Santander. Memorias y Resúmenes $44^{\circ}$ Congreso Socolen: Entomología de impacto Solución de problemas integrando metodologías. p 479. http://socolen.org.co/publicaciones/memorias-44-congresosocolen

SHARKEY, M. 1988. A taxonomic revision of Alabagrus (Hymenoptera: Braconidae). Bulletin of the British Museum (Natural History) Entomology 57: 311-437. http://biostor.org/ reference/113932

SOLIS, M.A.; METZ, M.A.; SCHEFFER, S.J.; LEWIS, M. L.; KULA, R. R.; SPRINGER, T. L. 2015. A new cryptic species of Diatraea (Lepidoptera: Crambidae: Crambinae) feeding on eastern gama grass and a novel host association with a braconid (Hymenoptera) in the United States. Annals of the Entomological Society of America 108 (4): 648-659. https://doi.org/10.1093/ aesa/sav049

SOLIS, M.A.; OSORIO-MEJÍA, P.; SARMIENTO-NAIZAQUE, Z. X.; BARRETO-TRIANA, N. 2020. A new species of Eoreuma Ely (Pyraloidea: Crambidae: Crambinae) feeding on sugarcane from Colombia. Proceedings of the Entomological Society of Washington 122 (2): 471-481. https://doi.org/10.4289/00138797.122.2.471

TARAZONA, G. 2011. Manejo fitosanitario del cultivo de caña panelera. Instituto Colombiano Agropecuario- ICA. Bogotá, D.C. Colombia. 48 p. https:/www.ica.gov.co/ getattachment/6a54658e-1723-488d-a7ab-2f4baad793cb/ Manejo-fitosanitario-del-cultivo-de-la-cana-panele.aspx
VARGAS, G. A.; OBANDO, V.; GÓMEZ, L. A. 2006. Jaynesleskia jaynesi: otra alternativa para el manejo de Diatraea spp. Carta Trimestral del Cenicaña 28 (2): 3-5. https://www.cenicana.org/ pdf_privado/carta_trimestral/ct2006/ct2_06/ct2_06_p3-5.pdf

VARGAS, G.; LASTRA, L.A.; SOLÍS, M.A. 2013. First record of Diatraea tabernella (Lepidoptera: Crambidae) in the Cauca River Valley of Colombia. Florida Entomologist 96 (3): 11981201. https://doi.org/10.1653/024.096.0367

VARGAS G.; GÓMEZ, L. A.; MICHAUD, J. P. 2015. Sugarcane stem borers of the Colombian Cauca River Valley: Current pest status, biology and control. Florida Entomologist 98 (2): 728735. https://doi.org/10.1653/024.098.0249

WEIR, E. H.; CONTRERAS, W.; GIL DE WEIR, K. 2007. Biological control of Diatraea spp. (Lep.: Pyralidae) in sugarcane crops in Central Venezuela. Revista de Biología Tropical 55 (2): 655-658. https://www.scielo.sa.cr/pdf/rbt/v55n2/3660.pdf

WHARTON, R. 1993. Bionomics of the Braconidae. Annual Review of Entomology 38: 121-143. https://doi.org/10.1146/annurev. en.38.010193.001005

WHITE, W. H.; REAGAN, T. E.; SMITH, J. A.; SALAZAR, J. 2004. Refuge releases of Cotesia flavipes (Hymenoptera: Braconidae) into the Louisiana sugarcane ecosystem. Environmental Entomology 33 (3): 627-632. https://doi.org/10.1603/0046225X-33.3.627

\section{Origen y financiación}

Este manuscrito fue originado en los proyectos de investigación "Fluctuación poblacional de Diatraea spp. y sus parasitoides en la Hoya del río Suárez" realizado entre 2015 y 2017 y "Estrategias de manejo integrado de plagas y enfermedades asociadas a cultivos de caña de azúcar en regiones productoras de panela en Colombia”, ambos financiados por el Ministerio de Agricultura y Desarrollo Rural de Colombia.

\section{Contribuciones de los autores}

Zaida Sarmiento: conceptualización, curaduría de datos, análisis formal, investigación, metodología, escritura (borrador original), escritura (correcciones del arbitraje y de edición); Carlos Sarmiento: conceptualización, análisis formal, investigación, metodología, validación, escritura (borrador original), escritura (correcciones del arbitraje y de edición); Nancy Barreto: conceptualización, análisis formal, adquisición de fondos, investigación, metodología, escritura (borrador original), escritura (correcciones del arbitraje y de edición).

\section{Conflictos de interés}

Los autores declaramos no tener conflictos de intereses. 